\title{
Phase-plate electron microscopy: a novel imaging tool to reveal close-to-life nano-structures
}

\author{
Kuniaki Nagayama $\cdot$ Radostin Danev
}

Received: 10 December 2008 / Accepted: 19 December 2008 / Published online: 17 January 2009

(C) The Author(s) 2009. This article is published with open access at Springerlink.com

\begin{abstract}
After slow progress in the efforts to develop phase plates for electron microscopes, functional phase plate using thin carbon film has been reported recently. It permits collecting high-contrast images of close-to-life biological structures with cryo-fixation and without staining. This report reviews the state of the art for phase plates and what is innovated with them in biological electron microscopy. The extension of thin-film phase plates to the material-less type using electrostatic field or magnetic field is also addressed.
\end{abstract}

Theoretically, electron microscopy is compatible with three different types of phase plate: thin-film, electrostatic, and magnetic. However, designing functional phase plates has been an arduous process that has suffered from unavoidable technical obstacles such as phase-plate charging and difficulties associated with micro-fabrication of electrostatic and magnetic phase plates. Firstly, this review introduces phase-contrast schemes able to visualize transparent objects usually unseen with conventional microscopic methods. Secondly, it deals with recent studies on biological applications ranging from proteins, viruses to cells. Thirdly, the key technology to overcome the obstacle of phase-plate

Electronic supplementary material The online version of this article (doi:10.1007/s12551-008-0006-z) contains supplementary material, which is available to authorized users.

K. Nagayama $(\bowtie) \cdot R$. Danev

Department of Physiological Science, SOKENDAI,

Okazaki Institute for integrative Bioscience,

National Institutes of Natural Sciences,

5-1 Higashiyama, Myodaiji cho,

Okazaki 444-8787, Japan

e-mail: nagayama@nips.ac.jp charging - the anti-static thin-film phase plate-is reported. Lastly, next generation phase plates to overcome the issue of electron loss inherent in the thin-film phase plates are discussed.

\section{Methodology of phase plates in electron microscopy}

The first phase visualization technique in optics was innovated at the end of the nineteenth century as Schlieren optics using the Foucault knife-edge, which halfly covers the back-focal plane of the objective lens (Fig. 1c). The Zernike phase contrast (ZPC; Fig. 1b) and the Smith/ Nomarski differential interference contrast (DIC) methods followed. In electron microscopy, instead, defocus phase contrast (DPC) was devised and developed by Scherzer which is now a standard in material electron microscopy (Fig. 1a; Scherzer 1949). The extension of ZPC to electron microscopy has been attempted for 60 years after the Boersch suggestion (Boersch 1947) but only recently one approach, using thin film phase plates, has been realized with substantial success (Danev and Nagayama 2001). This same group has also demonstrated a phase-plate version of DIC (Danev et al. 2002; Fig. 1d). The typical phase contrast, ZPC, and the oldest scheme, Schlieren optics, that is known in the electron microscopist community as singlesideband imaging, both can be realized with use of simple phase plates as shown in Fig. 1. However, the Smith/ Nomarski phase contrast, DIC, does not appear to be included in the simplest mode, as it employs a design to insert a birefringent optical device even before the objective lens. This methodological discord has been removed with a novel scheme that includes a semicircular phase-plate inserted at the back-focal-plane after the objective lens as a spatial filter to retard the electron waves by $\pi\left(180^{\circ}\right.$; 
Fig. 1 Various phase plates a A spatial filter schematic for the defocus phase contrast (DPC). b A spatial filter schematic for the Zernike phase contrast (ZPC). c A spatial filter schematic for Schlieren optics (SO) or singlesideband imaging (SSI). d A spatial filter schematic for Hilbert differential contrast (HDC)

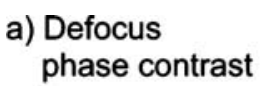

b) Zernike phase contrast d) Hilbert differential contrast

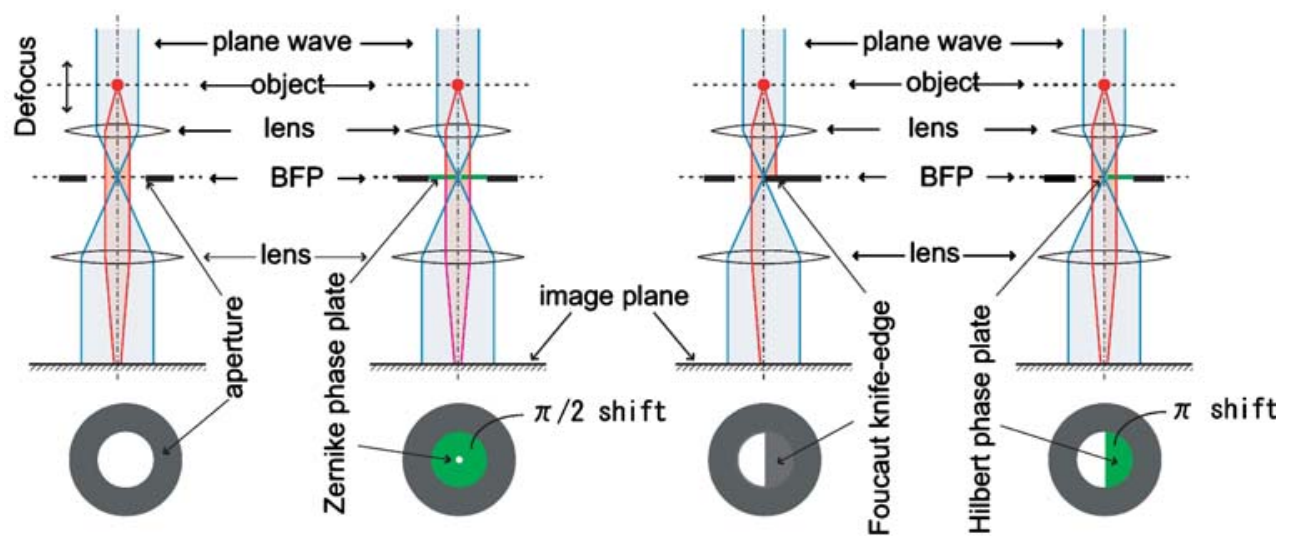

Danev et al. 2002). This setup was termed Hilbert differential contrast (Danev and Nagayama 2004).

Application of thin-film phase plates to biological samples

Cryo-technique The standard method of the sample preparation has been useful but also recognized troublesome by the destructive features using fixation, dehydration, resinembedding, staining, and thin-sectioning. The issues inherent in the traditional approach have all, but the image contrast been solved by cryo-techniques. Rapidly frozen ice-embedded specimens can provide close-to-life micrographic images as these samples are free from artifacts induced by the standard preparation method (Danev and Nagayama 2004; Van Harreveld and Crowell 1964; Heuser et al. 1979). This cryo-microscopy has been successfully utilized in electron cryo-tomography (Beck et al. 2007); (Deng et al. 2008) but not widely accepted partially because of costly instrumentation but mainly due to the low image contrast for unstained specimens.

Applications of Zernike phase-contrast TEM In ZPC, the finite size of the central hole limits the recoverable lower spatial frequencies. For a $300 \mathrm{kV}$ TEM system, for example, Zernike phase plate with a hole size of $0.5 \mu \mathrm{m}$ in diameter, results in a cut-on frequency about $0.05 \mathrm{~nm}^{-1}\left(20 \mathrm{~nm}^{-1}\right)$. Given this limitation, applications of ZPC-TEM can cover structural studies of relatively small biological systems.

Figure $2 \mathrm{a}$ and $\mathrm{b}$ illustrate the differences between $\mathrm{a}$ conventional and a ZPC image for a chaperonin protein, GroEL. Several competing groups intensively pursued high-resolution 3D structures of this protein with single particle analysis based on conventional images (Fig. 2b) (Ludtke et al. 2004; Saibil 2006; Stagg et al. 2006; Ludtke et al. 2008). The most difficult component of the single particle analysis when performed with the conventional approach is the first step, identification of the images that correspond to protein molecules. Images of ZPC have a great advantage in this particle identification as shown in (Fig. 2a; Danev and Nagayama 2008). The difference in the image contrast is theoretically rationalized by the difference in recoverable frequency components, which are characterized by image transfer processes with different contrast transfer functions (CTFs) defined in the Fourier space. Typical examples for CTFs, a sinusoidal form for the conventional and a cosinusoidal form for ZPC are inserted in Fig. $2 a$ and b. Contrast transfer functions can be experimentally determined by analyzing diffractograms as shown in the insets of Fig. $2 a$ and $b$.

Two other examples of ZPC images are shown in Fig. 2c and $\mathrm{d}$. One unresolved issue regarding endocytosis is the determinant for the membrane invagination. Many researchers have proposed that specific proteins mediate this process. Images obtained by phase-contrast cryo-TEM with a Zernike phase plate revealed that a recently discovered protein was associated with membrane invaginations (Shimada et al. 2007). Figure 2c shows a tubulate lipid membrane tightly wrapped by a filamentous polymer comprising a dimer of the protein, EFC/F-BA-domain. Xray crystallographic studies predicted that the spacing of the polymer wrapping around lipid is about $3.5 \mathrm{~nm}$, which fairly coincided with the spacing of $4.0 \mathrm{~nm}$ as experimentally determined (Fig. 2c).

Figure $2 \mathrm{~d}$ shows images for influenza A viruses in the iceembedded state (Yamaguchi et al. 2008). The glycoprotein spices and the lipid layers of the viral envelope were clearly resolved with high contrast and without CTF artifacts.

Application of Hilbert differential contrast TEM The most striking application of HDC-TEM to biology was the phase-contrast image of an entire cell in an ice-embedded state (Kaneko et al. 2005, 2006; Nitta et al. 2009). These results were completely unexpected in the TEM community, as whole-mounted cells have been considered too thick to be visualized without sectioning. The HDC-TEM images display topographic features and appear similar to images 
Fig. 2 TEM images $(300 \mathrm{kV})$ for proteins and viruses. a $\mathrm{A}$ ZPC image for an ice-embedded sample of GroEL (Danev and Nagayama 2008). The inset is a diffractogram obtained by Fourier-transform of the image. A typical cosine CTF is shown. This can be experimentally determined with the kind of diffractogram shown in the inset. $\mathbf{b}$ A conventional image for an ice-embedded sample of GroEL (Danev and Nagayama 2008). The inset is a diffractogram obtained by Fourier-transform of the image. A typical sine CTF is shown. This can be experimentally determined with the kind of diffractogram shown in the inset. c A ZPC image for a tubulate membrane liposome wrapped by a polymeric protein fiber (Shimada et al. 2007). d A ZPC image of ice-embedded influenza A viruses (Yamaguchi et al. 2008)
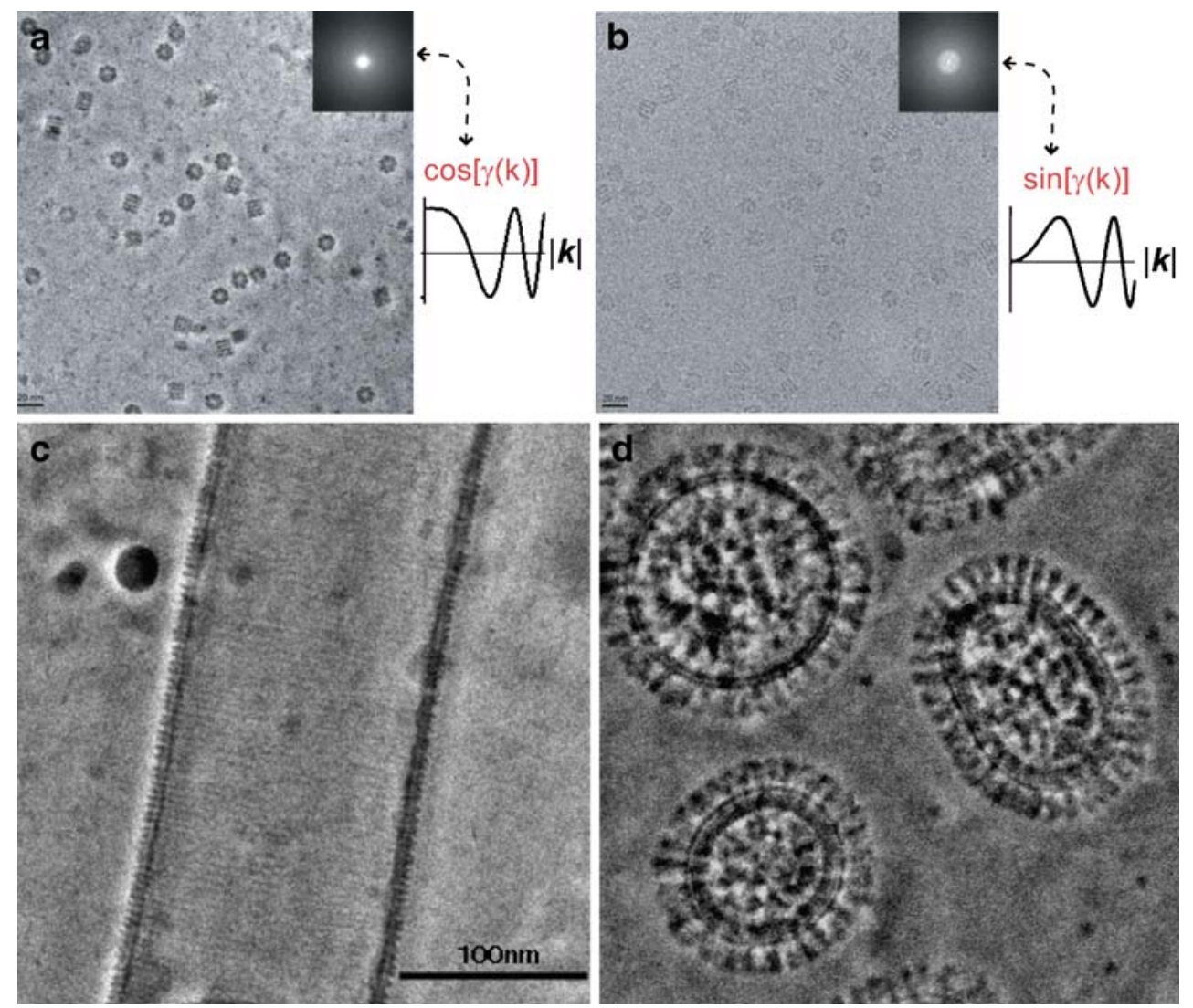

obtained with differential interference contrast light microscopy (Danev et al. 2002) as shown in Fig. 3 (Kaneko et al. 2005). Surrounded by smooth cell walls, the thylakoid membranes and carboxysomes are visible inside the micrograph of HDC (Fig. 3a). These structural features were compared with micrographs obtained with conventional TEM images of ultrathin sections of chemically fixed and resin-embedded cyanobacteria (Fig. 3c). What was firstly recognized was the empty holes in the conventional image as shown by an arrow in Fig. 3c. They were attributed
Fig. 3 TEM images $(300 \mathrm{kV})$ of a vitrified cyanobacterial cell. a A HDC just-focused image of an ice-embedded cyanobacterial whole cell (Kaneko et al. 2005). The polyphosphate bodies (shown by an arrow) are prominent among detailed ultrastructures. b A conventional TEM image of an ice-embedded cyanobacterial cell for the same view field as a defocus setting of $15 \mu \mathrm{m}$ (Kaneko et al. 2005). c A $100 \mathrm{kV}$ conventional TEM image of a chemically fixed, resinembedded, and thin-sectioned cell stained with uranyl acetate and lead citrate (Kaneko et al. 2005). Polyphosphate bodies are lost during the preparation process. This leaves an empty hole in the section (arrow) (Kaneko et al. 2005)
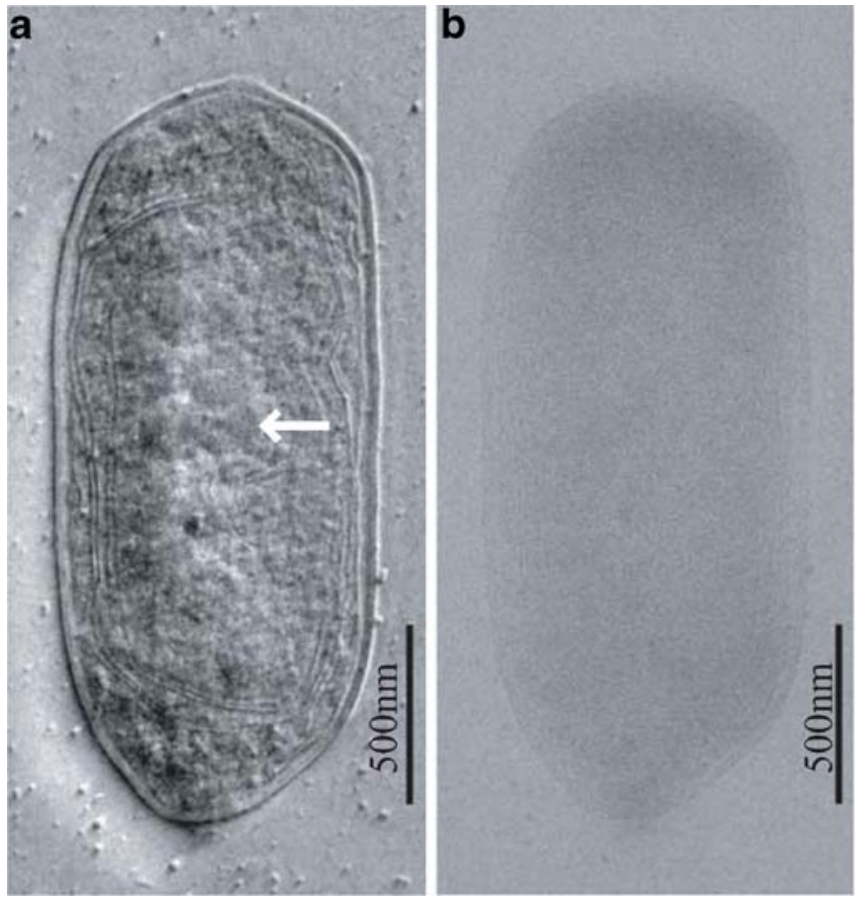


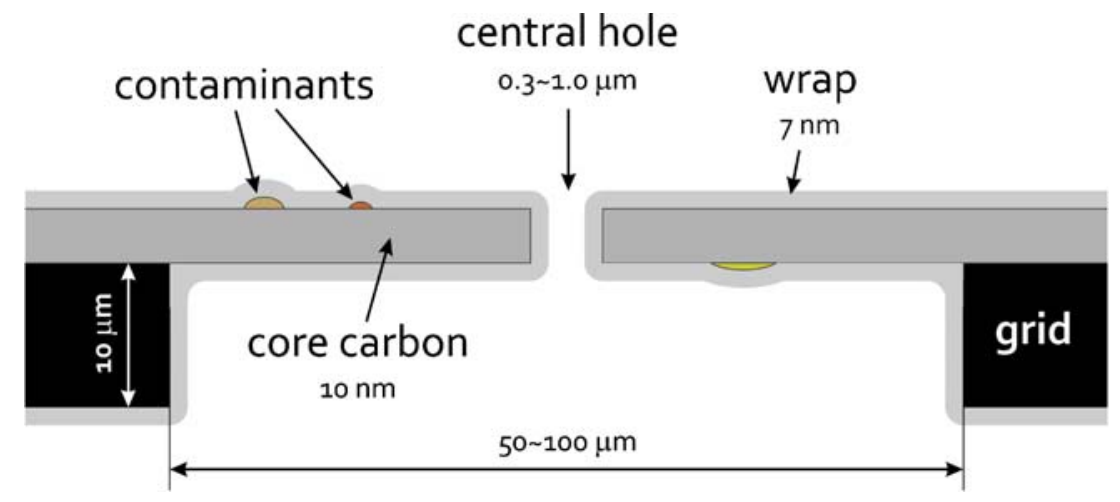

Fig. 4 A three-layered carbon film design to avoid the charging effect in a Zernike phase plate (Nagayama 2005). Contaminants are wrapped by a conductive carbon coat to shield the electrostatic potential

to the vacancy of spherical polyphosphate bodies, which were clearly visible with ZPC as electron dense objects as shown by the arrow in Fig. 3a. Contrary to these two images, fine structures inside the cell are almost invisible in the image for ice-embedded unstained cells obtained with the conventional method (Fig. 3b).

\section{Workable phase plates made of carbon thin films}

TEM pioneers noted that contamination on the surface of phase plates by insulating materials was the source of the charge. The phase plate itself is not charged when made of a conducting material such as carbon. Efforts for hunting after actual origins of contaminants clarified that there were three sources for charged contaminants: organic materials, metal oxides, and inorganic materials, which were unavoidably integrated into or onto phase plates during the fabrication procedure (Nagayama 2005). For the final answer to settle the dilemma of fabricating or not fabricating, they employed an old idea-electrostatic shielding. The charge-induced potential can be shielded by wrapping charges with conductive material such as carbon. In the final step of phase-plate production, either sides of the phase plate, likely contaminated with organic materials, metal oxides, or inorganic materials, are coated with carbon in a vacuum evaporator. Consequently, once grounded, the electrostatic potential arising from charges is eliminated. The schematic of a three-layered phase plate constructed with a carbon wrapping finish is shown in Fig. 4.
Fig. 5 a An electrostatic phase plate made of a five-layered electrode supported by three spokes (Majorovits et al. 2007). b An electrostatic phase plate made of a double-layered electrode supported by a single spoke (Cambie et al. 2007). c An example of a five-bar magnet $(0.8 \mu \mathrm{m}$ width and $2 \mathrm{~mm}$ length) made of cobalt thin-film deposited on a biprism (Nagayama 2008). d A schematic of an anamorphotic phase plate. The spit-shaped device has embedded electrodes for application of electrostatic fields to a high aspect ratio anamorphotic diffraction plane electron wave (Schröder et al. 2007)
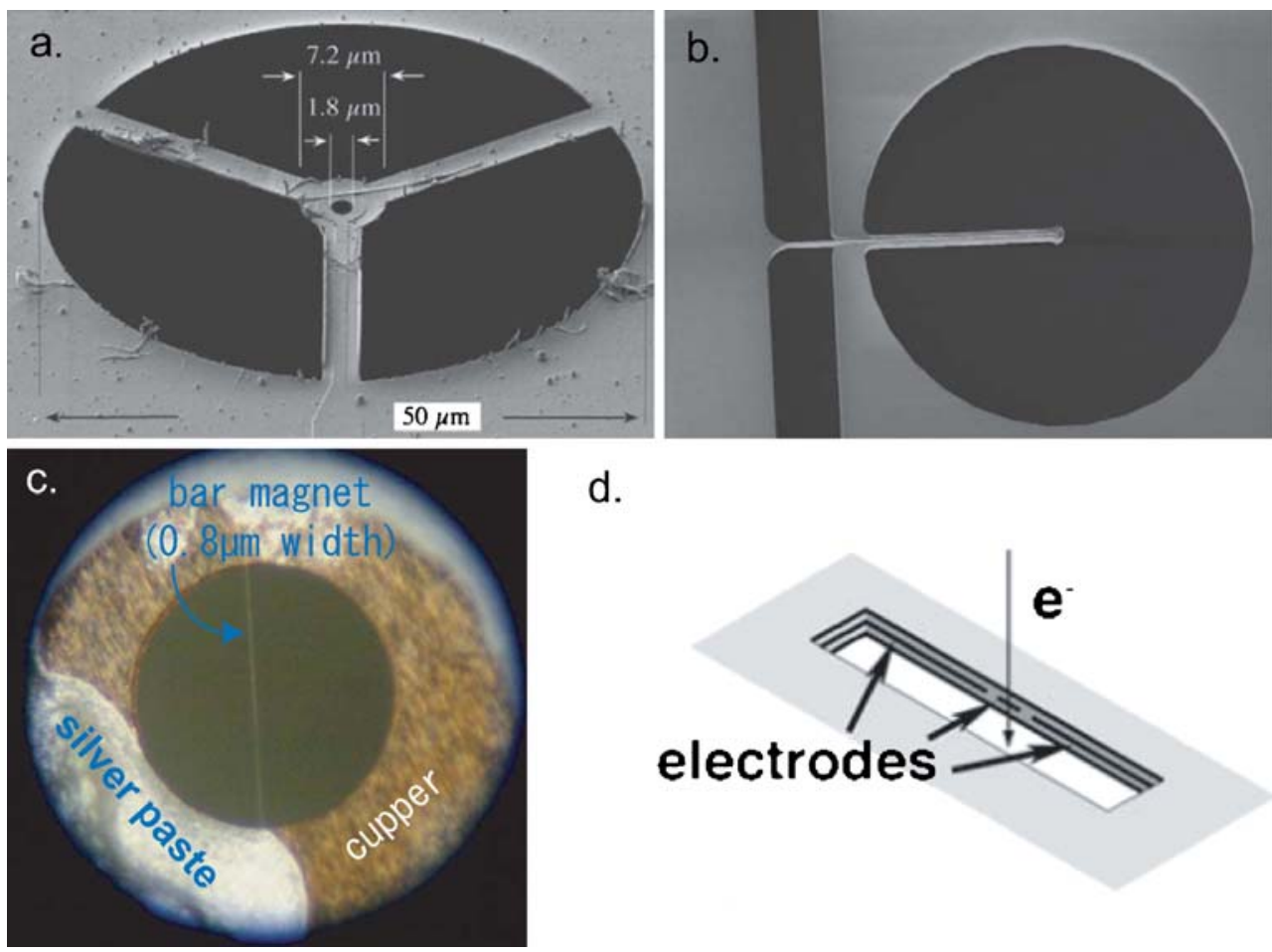

d.

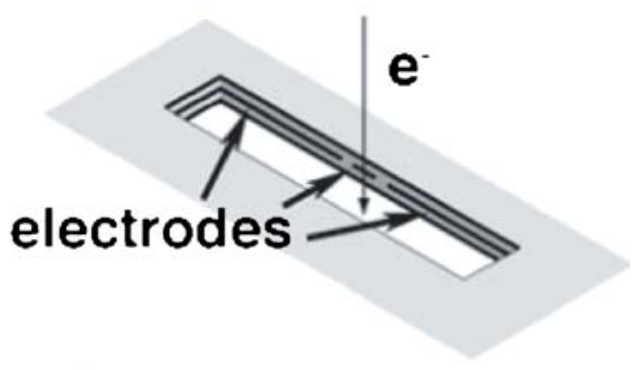




\section{Next-generation phase plates}

One of the disadvantages in using thin-film phase-plates is electron loss due to electron scattering (Danev and Nagayama 2001). In his pioneering work, Boersch proposed other options for phase plates - electrostatic potential type. The sophisticated version of the Zernike phase plate containing a static ring electrode that can arbitrarily control the amount of phase shift was firstly proposed as the numerical work (Matumoto and Tonomura 1996) and recently three groups have reported that the electrostatic phase plate is technically tractable with the use of advanced microfabrication technology (Majorovits et al. 2007; Cambie et al. 2007; Huang et al. 2006). All three reports confirmed that the phase shift by $\pi / 2$ corresponds to ZPC, but efficient phase contrast has yet to be reported in part due to the large outer diameter of the rings blocking the lower frequency components (remember $0.5-1.0 \mu \mathrm{m}$ in diameter for the hole of the Zernike phase plate with thin films). In the design of these electrostatic phase plates, except for the Berkeley group, insulators prone to be changed are utilized to separate electrodes.

In addition to electrostatic potential, another factor that can affect the electron phase is vector potential. The AharonovBohm (AB) effect emerged as a test to prove the reality of the vector potential (Aharonov and Bohm 1959). A decisive answer to this test was provided by the Hitachi group by using a small permalloy ring perfectly shielded with a superconducting material (Tonomura et al. 1986). In connection with this elegant result, the Hitachi group proposed to use a small permalloy ring to shift the phase of the incident electrons by $\pi / 2$, while demands to fabricate the Zernike AB phase plate seemed to be a technical blockage.

Recently, the Nagayama group offered a solution to overcome the technical difficulty. They proposed applying the $\mathrm{AB}$ effect to the Hilbert phase plate to control the extent of the phase change desired for HDC. One of the examples using a thin bar magnet is shown in Fig. 5c, which has desirable characteristics such as very small electronintercepting area and an exclusive usage of conducting materials (Nagayama 2008).

Another type of material-less phase plate which was proposed recently is the anamorphotic design (Schröder et al. 2007). Figure 5 b shows a schematic of the slit-like phase plate which uses electrostatic fields and must be positioned at an anamorphotic diffraction plane. Such a plane has different magnifications in $x$ and $y$ directions producing a high aspect ratio stretched diffraction pattern that must be oriented along the slit of the phase plate. The realization of this type of phase plate depends mainly on the ability to produce the anamorphotic plane. It will require complicated multiple multipole optical system, similar to the ones found in Cs and Cc correctors, and has yet to be realized and tested.
Open Access This article is distributed under the terms of the Creative Commons Attribution Noncommercial License which permits any noncommercial use, distribution, and reproduction in any medium, provided the original author(s) and source are credited.

\section{References}

Aharonov Y, Bohm D (1959) Significance of electromagnetic potentials in the quantum theory. Phys Rev 115:485-491

Beck M, Lučić V, Förster F, Baumeister W, Medalia O (2007) Snapshots of nuclear pore complexes in action captured by cryoelectron tomography. Nature 449:611-615

Boersch H (1947) Über die Kontraste von Atomen in Electronenmikroskop. Z Naturforschg 2a:615-633

Cambie R, Downing KH, Typke D, Glaeser RM, Jin J (2007) Design of a microfabricated, two-electrode phase-contrast element suitable for electron microscopy. Ultramicroscopy 107:329339

**Danev R, Nagayama K (2001) Transmission electron microscopy with Zernike phase plate. Ultramicroscopy 88:243-252 doi:10.1016/S0304-3991(01)00088-2

Danev R, Nagayama K (2004) Complex observation in electron microscopy. IV. Reconstruction of complex object wave from conventional and half plane phase plate image pair. J Phys Soc Jpn 73:2718-2724

*Danev R, Nagayama K (2008) Single particle analysis based on Zernike phase contrast transmission electron microscopy. J Struc Biol 161:211-218

**Danev R, Okawara H, Usuda N, Kametani K, Nagayama K (2002) A novel phase-contrast transmission electron microscopy producing high-contrast topographic images of weak objects. J Biol Phys 28:627-635

Deng B, O'Connor CM, Kedes DH, Zhou ZH (2008) Cryo-electron tomography of Kaposi's sarcoma-associated herpesvirus capsids reveals dynamic scaffolding structures essential to capsid assembly and maturation. J Struct Biol 161:419-427

Heuser JE, Reese TS, Dennis MJ, Jan Y, Jan L, Evans L (1979) Synaptic vesicle exocytosis captured by quick freezing and correlated with quantal transmitter release. J Cell Biol 81:275-300

Huang SH, Wang WJ, Chang CS, Hwu YK, Kai JJ, Chen FR (2006) The fabrication and application of Zernike electrostatic phase plate. J Electron Mirosc 55:273-280

**Kaneko Y, Danev R, Nitta K, Nagayama K (2005) In vivo subcellular ultrastructures recognized with Hilbert-differentialcontrast transmission electron microscopy. J Electron Microsc 54:79-84

Kaneko Y, Danev R, Nagayama K, Nakamoto H (2006) Intact carboxysome in a cyanobacterial cell visualized by Hilbert differential contrast transmission electron microscopy. J Bacteriol 188:805-808

Ludtke SJ, Chen DH, Song JL, Chuang DT, Chiu W (2004) Seeing GroEL at $6 \mathrm{~A}^{\circ}$ resolution by single particle electron cryomicroscopy. Structure 12:1129-1136

*Ludtke SJ, Baker ML, Chen DH, Song JL, Chuang DT, Chiu W (2008) De novo backbone trace of GroEL from single particle electron cryomicroscopy. Structure 16:441-448

Majorovits E, Barton B, Schultheiß K, Perez-Willard F, Gerthsen D, Schröder RR (2007) Optimizing phase contrast in transmission electron microscopy with an electrostatic (Boersch) phase plate. Ultramicroscopy 107:213-226

Matumoto T, Tonomura A (1996) The phase constancy of electron waves traveling through Boersch's electrostatic phase plate. Ultramicroscopy 63:5-10 
*Nagayama K (2005) Phase contrast enhancement with phase plates in electron microscopy. Ad Imaging Electr Phys 138:69-146

Nagayama K (2008) Development of phase plates for electron microscopes and their biological application. Eur Biophys J 37:345-358

Nitta K, Kaneko Y, Nagayama K (2009) Visualization of BrdU incorporating DNA in cyanobacterial cells by Hilbert differential contrast transmission electron microscopy. J. Microscopy in press

Saibil HR (2006) Allosteric signaling of ATP hydrolysis in GroELGroES complexes. Nat Struct Mol Biol 13:147-152

Scherzer O (1949) The theoretical resolution limit of the electron microscope. J Appl Phys 20:20-29 doi:10.1063/1.1698233

Schröder RR, Barton B, Rose H, Genner G (2007) Contrast enhancement by anamorphotic phase plates in an aberration corrected TEM. Microsc Microanal 13:136-137

*Shimada A, Niwa H, Tsujita K, Suetsugu S, Nitta K, Suetsugu KH, Akasaka R, Nishino Y, Toyama M, Chen L, Liu ZJ, Wang BC, Yamamoto M, Terada T, Miyazawa A, Tanaka A, Sugano S, Shirouzu M, Nagayama K, Takenawa T, Yokoyama S (2007) Curved $\mathrm{EFC} / \mathrm{F}-\mathrm{BAR}$-domain dimers are joined end to end into a filament for membrane invagination in endocytosis. Cell 129:761-772

Stagg SM, Lander GC, Pulokas J, Fellmann D, Cheng A, Qulspe JD, Mallick SP, Avila RM, Crragher B, Potter CS (2006) Automated cryoEM data acquisition and analysis of 284742 particles of GroEL. J Struct Biol 155:470-481

Tonomura A, Osakabe N, Matsuda T, Kawasaki T, Endo J, Yano S, Yamada H (1986) Evidence for Aharonov-Bohm effect with magnetic field completely shielded from electron wave. Phys Rev Lett 56:792-795

Van Harreveld A, Crowell J (1964) Electron microscopy after rapid freezing on a metal surface and substitution fixation. Anat Rec 149:381-386

*Yamaguchi M, Danev R, Nishiyama K, Sugawara K, Nagayama K (2008) Zernike phase contrast electron microscopy of iceembedded influenza A virus. J Struct Biol 162:271-276

This work was partially supported by CREST (Core Research for Evolutional Science and Technology) of JST (Japan Science and Technology Agency).
**Danev and Nagayama 2001. The first report in the last 30 years of a successful implementation of a thin-film Zernike phase plate in TEM. A comparison of images of negatively stained ferritin demonstrates the improvement brought by the phase plate. Contains analysis of different materials for phase-plate preparation and discussion about the effects of electrostatic charging and beam induced contamination.

**Danev et al. 2002. Introduction of the Hilbert differential contrast. Contains theoretical analysis of the contrast transfer and point spread functions. Experimental examples using plastic embedded tissue sections demonstrate the ability of the Hilbert imaging mode to produce high-contrast images containing many details.

*Ludtke et al. 2008. A report of the backbone tracing of the chaperonin GroEL based on a 4A density map produced by conventional single particle electron cryo-microscopy.

*Danev and Nagayama 2008. Presents the first application of Zernike phase contrast to single particle analysis. The results using ice-embedded GroEL show about 30\% improvement in efficiency ( $30 \%$ less particles are needed to achieve the same resolution) in favor of the Zernike phase contrast compared to conventional TEM.

* Shimada et al. 2007. Application of Zernike phase contrast for the observation of a morphological conversion process of liposomes from a spherical to a tubular form in a model system of endocytosis. Protein filaments made of EFC dimmers are visualized to wind liposomes tightly to squeeze them for membrane invagination.

*Yamaguchi et al. 2008. Investigation of the influenza A virus using Zernike phase-contrast cryo-microscopy. The representative images have high contrast and contain enough detail for direct interpretation of virion features such as shape, number and spacing of lipid layers and distribution of envelope spikes.

**Kaneko et al. 2005. Application of Hilbert differential contrast for the observation of ice-embedded whole cell cyanobacteria. Many details and features inside the cell are revealed by the improved contrast and information transfer.

* Nagayama 2005. A review article to summarize the recent development of thin-film phase plates applicable to two kinds of phase-contrast methods, Zernike phase contrast and Hilbert differential contrast, in electron microscopy. Various applications of both phase-contrast methods to biological systems such as proteins, viruses, bacteria and cells are also reviewed. 